\title{
Grassmannian-parameterized solutions to direct-sum polygon and simplex equations
}

\author{
Aristophanes Dimakis Igor Korepanov
}

October 2020

\begin{abstract}
We consider polygon and simplex equations, of which the simplest nontrivial examples are pentagon (5-gon) and Yang-Baxter (2-simplex), respectively. We examine the general structure of $(2 n+1)$-gon and $2 n$ simplex equations in direct sums of vector spaces. Then we provide a construction for their solutions, parameterized by elements of the Grassmannian $\operatorname{Gr}(n+1,2 n+1)$.
\end{abstract}

\section{Introduction}

\subsection{Generalities about set-theoretic polygon and simplex equations}

Polygon and simplex equations are two families of algebraic equations that naturally arise, and find applications, in mathematical physics, topology, representation theory; there is also interesting combinatorics related to these equations. A short but comprehensive enough historical review, with many references, can be found in the Introduction to paper [4]; here we just mention some aspects of our interest in the present paper.

The first nontrivial example of polygon equations is pentagon equation

$$
A_{12}^{(1)} A_{13}^{(3)} A_{23}^{(5)}=A_{23}^{(4)} A_{12}^{(2)},
$$

below we also represent it graphically in Figure 1. Concerning the superscripts in (11), we hope to explain below why we choose them like that; right now it is enough to say that they symbolize that $A^{(q)}$ with different $q$ 's may be different objects.

In the "set-theoretic" interpretation of (11), the subscripts represent the sets where mappings $A^{(q)}$ act. Usually, these sets carry some algebraic structure, for instance, of a group or a quandle (the latter also called "distributive 
groupoid" [18, 9]). In (11), three sets $X_{1}, X_{2}$ and $X_{3}$ are involved; often-but not

necessarily - they are copies of one fixed set $X$. Each mapping $A_{i j}^{(q)}$ acts in the corresponding direct product:

$$
A_{i j}^{(q)}: \quad X_{i} \times X_{j} \rightarrow X_{i} \times X_{j} .
$$

Also, $A_{i j}^{(q)}$ is identified with the direct product of itself and the identity mapping $\mathbb{1}_{k}$ in the lacking set $X_{k}$, so the whole l.h.s. and r.h.s. of (11) act in $X_{1} \times X_{2} \times X_{3}$.

Similarly, the first nontrivial example of simplex equations is the Yang-Baxter equation

$$
R_{12}^{(1)} R_{13}^{(2)} R_{23}^{(3)}=R_{23}^{(3)} R_{13}^{(2)} R_{12}^{(1)}
$$

then goes Zamolodchikov tetrahedron equation,

$$
R_{123}^{(1)} R_{145}^{(2)} R_{246}^{(3)} R_{356}^{(4)}=R_{356}^{(4)} R_{246}^{(3)} R_{145}^{(2)} R_{123}^{(1)},
$$

then "4-simplex equation", and so on. In the set-theoretic setting, they are interpreted in the same way as described above. For instance, in (3), each $R$ maps the direct product of three sets into itself:

$$
R_{i j k}^{(q)}: \quad X_{i} \times X_{j} \times X_{k} \rightarrow X_{i} \times X_{j} \times X_{k},
$$

and is also identified when necessary with its direct product with the identity mappings in the lacking sets.

There are many interrelations between polygon and simplex equations. Of these, we mention here, and will exploit in this paper, the "three-color decomposition" of simplex equations. This means that, assuming a special form (see (28) below) of solutions, the simplex equation breaks into three independent parts, two of which are nothing but polygon equations, while the third can be treated as a "consistency condition" between these two. Such decomposition first appeared, for pentagon and 4-simplex, in [11], and was generalized to other equations and analyzed combinatorially in detail in [4]. In some cases, it allows to construct simplex solutions from polygon solutions.

\subsection{Direct-sum equations and motivation for their study}

Our "direct-sum" equations are a particular case of set-theoretic equations. Namely, each $X_{i}=V_{i}$ is a vector space over a field $F$; accordingly $A_{i j}^{(q)}: V_{i} \oplus V_{j} \rightarrow$ $V_{i} \oplus V_{j}$ are linear operators, and everything happens in the direct sum of spaces $V_{i}$. Field $F$ is allowed to be finite, as well as all $V_{i}$.

Direct-sum Yang-Baxter equations have been used in knot theory for quite a long time, although not necessarily under this name. Namely, the famous tricolorability [7, p. 8] property (not to be confused with the three-color decomposition dealt with below in this paper!) that allows to distinguish the trefoil knot from 
the unknot uses the rule of coloring knot fragments that can be described in terms of the direct-sum Yang-Baxter, with the field $F=\mathbb{F}_{3}$ being the Galois field of three elements. More generally, the Alexander quandle [9], considered over field $F$, gives a coloring rule of a direct-sum Yang-Baxter kind, which can be written as

$$
\left(\begin{array}{ll}
x & y
\end{array}\right) \mapsto\left(\begin{array}{ll}
x & y
\end{array}\right)\left(\begin{array}{cc}
1 & 1-t \\
0 & t
\end{array}\right),
$$

where $t \in F$ is a parameter (note that we thus prefer in this paper to regard matrices as acting on rows rather than columns).

Similarly to how Yang-Baxter is applied to knot theory, set-theoretic-and in particular direct-sum-solutions to polygon equations can be expected to find their applications to piecewise linear manifold invariants. That is because they correspond naturally to Pachner moves - elementary re-buildings of a triangulation of a given manifold. For instance, pentagon corresponds to Pachner move $2-3$ in three dimensions.

Returning to Yang-Baxter, one more interesting area where its direct-sum solutions have appeared is the tropical limit of Kadomtsev-Petviashvili and Korteweg-deVries solitonic equations, see [5, formulas (5.6), (5.7)].

More general direct-sum simplex equations - generalizations of YangBaxter - appeared in Hietarinta's paper [8], where they were used for constructing "permutation-type" solutions to quantum equations. Permutation-type solutions can be expected to be the first step in constructing more sophisticated solutions to higher simplex equations.

\subsection{Constant and nonconstant solutions}

Sets $X_{i}$ introduced above are often naturally isomorphic to one another, that is, $X_{1}, X_{2}, \ldots$ are just copies of one set $X$; the same applies of course to vector spaces $V_{1}, V_{2}, \ldots$ This allows us to speak of the case where our mappings, or linear operators, such as $A^{(q)}$ or $R^{(q)}$, are also copies of one and the same mapping/operator for all $q$ 's. In this case we call the considered equation constant. Constant equations are certainly of interest: enough to say that it is exactly the constant Yang-Baxter that finds its applications in knot theory and its generalizations [3] (the same applies to the quantum case [1]). Also, Hietarinta in his paper [8] classified solutions to constant Yang-Baxter and higher simplex equations.

If mappings/operators are allowed to be different, the equation is called nonconstant. We said already that this is exactly the case in our equations such as (11) or (2). So, our paper is about the non-constant case. 


\subsection{What is done in the main part of the paper}

Speaking more specifically about our linear operators, they will depend on parameters that can be thought of together as an element of the Grassmannian $\operatorname{Gr}(n+1,2 n+1)$ - an $(n+1)$-dimensional plane in a $(2 n+1)$-dimensional linear space, $n=1,2, \ldots$ This construction provides solutions to $(2 n+1)$-gon and $2 n$-simplex equations. In the trivial but still instructive case $n=1$, these are trigon and Yang-Baxter, see Example 8 below; for $n=2$, these are pentagon and 4-simplex, and so on. All our solutions depend only on ratios of Plücker coordinates of the Grassmannian, see (44) and (45) below. Our "polygons" here have thus always an odd number $(2 n+1)$ of "vertices", so we leave for future work half of the possible equations; no doubt that they are interesting and deserve a separate research.

Below, in Section 2, we investigate the general structure of $(2 n+1)$-gon and $2 n$-simplex equations in direct sums of vector spaces. In particular, we describe the three-color decomposition [4] of the $2 n$-simplex equations that permits in some cases to obtain their solutions from solutions of $(2 n+1)$-gon equations. Although we do it for our direct-sum case, the reader can notice that the combinatorial structure will be the same for either general set-theoretical or quantum case. We show also how to obtain solutions to $(2 n-1)$-simplex equations using a reduction procedure.

Then, in Section 3, we provide our Grassmannian-based solutions. The proof that they are indeed solutions for any $n$ combines Grassmann algebra and Plücker bilinear relations.

Finally, in Section 4, we discuss our results and possible directions for further research.

\section{$2(2 n+1)$-gon and $2 n$-simplex equations in di- rect sums}

\subsection{General notions}

Polygon and simplex equations in direct sums of vector spaces are equations on linear operators. Denote these spaces, for a chosen equation, $V_{i}$, where index $i$ runs over some (finite) set $\mathcal{C} \ni i$. The equation lives hence in

$$
V_{\mathcal{C}} \stackrel{\text { def }}{=} \bigoplus_{i \in \mathcal{C}} V_{i} .
$$

Each individual operator, however, acts nontrivially only in some spaces. That is, for such operator $A$ there is a subset $\mathcal{B} \subset \mathcal{C}$ such that both spaces $V_{\mathcal{B}}$ and $V_{\mathcal{C} \backslash \mathcal{B}}$ (defined in the same way as in (44)) are invariant for $A$ and, moreover, 
$A$ acts as an identity operator in $V_{\mathcal{C} \backslash \mathcal{B}}$ :

$$
A=\left.A\right|_{\mathcal{B}} \oplus \mathbb{1}_{\mathcal{C} \backslash \mathcal{B}} .
$$

In this situation, we identify $A$ with its restriction $\left.A\right|_{\mathcal{B}}$.

In this paper, we consider the simplest case where each $V_{i}$ is a one-dimensional space over a field $F$, and has a fixed basis. This means of course that we have identified each $V_{i}$ with $F$, and also our operators are naturally identified with matrices.

\section{$2.2(2 n+1)$-gon equation}

The direct sum $(2 n+1)$-gon equation considered here is defined on a vector space $V_{(2 n+1) \text {-gon }}$ of dimension $\left(\begin{array}{l}n \\ 2\end{array}\right)=\frac{n(n+1)}{2}$ over a field $F$. We think of it, in accordance with Subsection 2.1, as a direct sum of one-dimensional spaces $V_{i}$, where $i$ takes values in the set

$$
\mathcal{C}=[n(n+1) / 2] \stackrel{\text { def }}{=}\{1,2, \ldots, n(n+1) / 2\} .
$$

Explicitly, we write elements of $V_{(2 n+1) \text {-gon }}$ as $\frac{n(n+1)}{2}$-row vectors, and the $(2 n+1)$ gon equation is

$$
A_{\mathcal{B}_{1}}^{(1)} A_{\mathcal{B}_{3}}^{(3)} \cdots A_{\mathcal{B}_{2 n+1}}^{(2 n+1)}=A_{\mathcal{B}_{2 n}}^{(2 n)} A_{\mathcal{B}_{2 n-2}}^{(2 n-2)} \cdots A_{\mathcal{B}_{2}}^{(2)} .
$$

Here each $A^{(q)}, \quad q=1,2, \ldots, 2 n+1$, is a linear operator acting nontrivially in the direct sum of $n$ one-dimensional spaces and thus identified, according to Subsection 2.1, with an $n \times n$-matrix; as our space $V_{(2 n+1) \text {-gon }}$ consists of row vectors, the matrices act on the right. Each $\mathcal{B}_{q}$ in (5) is the set of numbers of spaces, or simply positions in the row where $A^{(q)}$ acts nontrivially:

$$
\mathcal{B}_{q}=\left\{b_{q, 1}, \ldots, b_{q, n}\right\} .
$$

Below, we are going to specify these $\mathcal{B}_{q}$ in a way that may seem somewhat roundabout, but is actually very convenient for our aims here.

Convention 1. In this paper, we list the elements of all sets always in the increasing order, if not explicitly stated otherwise. For instance, $b_{q, 1}<\cdots<b_{q, n}$ in (6).

We introduce two sequences of pairs of numbers from $\{1,2, \ldots, 2 n+1\}$ called initial and final sequence. Initial sequence consists of pairs (odd number, even number), taken in the lexicographic order, that is,

$$
\begin{aligned}
(1,2),(1,4), \ldots,(1,2 n), \ldots \\
\quad(\mathbf{2} \boldsymbol{k}-\mathbf{1}, \mathbf{2} \boldsymbol{k}), \ldots,(\mathbf{2} \boldsymbol{k}-\mathbf{1}, \mathbf{2 n}), \ldots,(2 n-1,2 n) .
\end{aligned}
$$


Here we highlighted in bold a "typical" subsequence consisting of pairs containing $2 k-1$. Similarly, final sequence consists of pairs (even number, odd number), taken also in the lexicographic order, that is,

$$
\begin{aligned}
&(2,3),(2,5), \ldots(2,2 n+1), \ldots, \\
&(\mathbf{2} \boldsymbol{k}, \mathbf{2} \boldsymbol{k}+\mathbf{1}), \ldots,(\mathbf{2} \boldsymbol{k}, \mathbf{2 n}+\mathbf{1}), \ldots,(2 n, 2 n+1),
\end{aligned}
$$

where we highlighted in bold a "typical" subsequence consisting of pairs containing $2 k$. Both sequences clearly have length $\frac{n(n+1)}{2}$.

By definition, $\mathcal{B}_{q}$ consists of such positions $b=b_{q, i}$ for which $q$ is present in the $b$ 's pair at least in one of the sequences (7) and (8). Clearly, there are exactly $n$ such positions for any $q=1,2, \ldots, 2 n+1$. Explicit formulas for $\mathcal{B}_{q}$ are given in Subsection 2.4.

We introduce the following indexing rule for input and output $n$-rows for matrices $A^{(q)}$. For a given $q$, define the set

$$
L_{q}=\{1,2, \ldots, 2 n+1\} \backslash\{q\} \stackrel{\text { def }}{=}\left\{p_{1}, p_{2}, \ldots, p_{2 n}\right\},
$$

then the entries of the mentioned rows are indexed as follows:

$$
\left(\begin{array}{llll}
u_{p_{1}, q} & u_{p_{3}, q} & \ldots & u_{p_{2 n-1}, q}
\end{array}\right) A^{(q)}=\left(\begin{array}{llll}
u_{p_{2}, q} & u_{p_{4}, q} & \ldots & u_{p_{2 n}, q}
\end{array}\right) .
$$

Here we identify if needed $\left(p_{i}, q\right)$ with $\left(q, p_{i}\right)$, this cannot bring confusion.

Proposition 1. The indexing rule (10) is consistent with both sides of the $(2 n+$ 1)-gon equation (5). Namely, take an input row vector for both sides of (5) whose entries are indexed according to the sequence (7):

$$
\left(\begin{array}{llll}
u_{1,2} & u_{1,4} & \ldots & u_{2 n-1,2 n}
\end{array}\right) .
$$

Then, as we apply in turn the matrices $A^{(q)}$ in either l.h.s. or r.h.s. of (5) and change at each step the indexing of the corresponding $n$ entries according to (10),

(i) at each step, there are exactly $n$ index pairs in the input $\frac{n(n+1)}{2}$-row fitting (10),

(ii) the final obtained sequence coincides with (8).

Proof. (i) Indeed, consider the l.h.s. for concreteness. There are of course exactly $n$ index pairs in (11) fitting $A^{(1)}$. After applying $A^{(1)}$, there appear $u_{1,3}, u_{1,5}, \ldots$, so, in particular, there are now $n$ index pairs fitting $A^{(3)}$, and so on.

(ii) In the 1.h.s., an entry $u_{2 k-1,2 l}$ is first replaced with $u_{2 k-1,2 l+1}$ by $A^{(2 k-1)}$ and then with $u_{2 k, 2 l+1}$ by $A^{(2 l+1)}$, as required. In the r.h.s., an entry $u_{2 k-1,2 l}$ is first replaced with $u_{2 k, 2 l}$ by $A^{(2 l)}$ and then with $u_{2 k, 2 l+1}$ by $A^{(2 k)}$, except for the case $k=l$, where only one matrix, $A^{(2 k)}$, takes part in the play, and replaces $u_{2 k-1,2 k}$ directly with $u_{2 k, 2 k+1}$. 

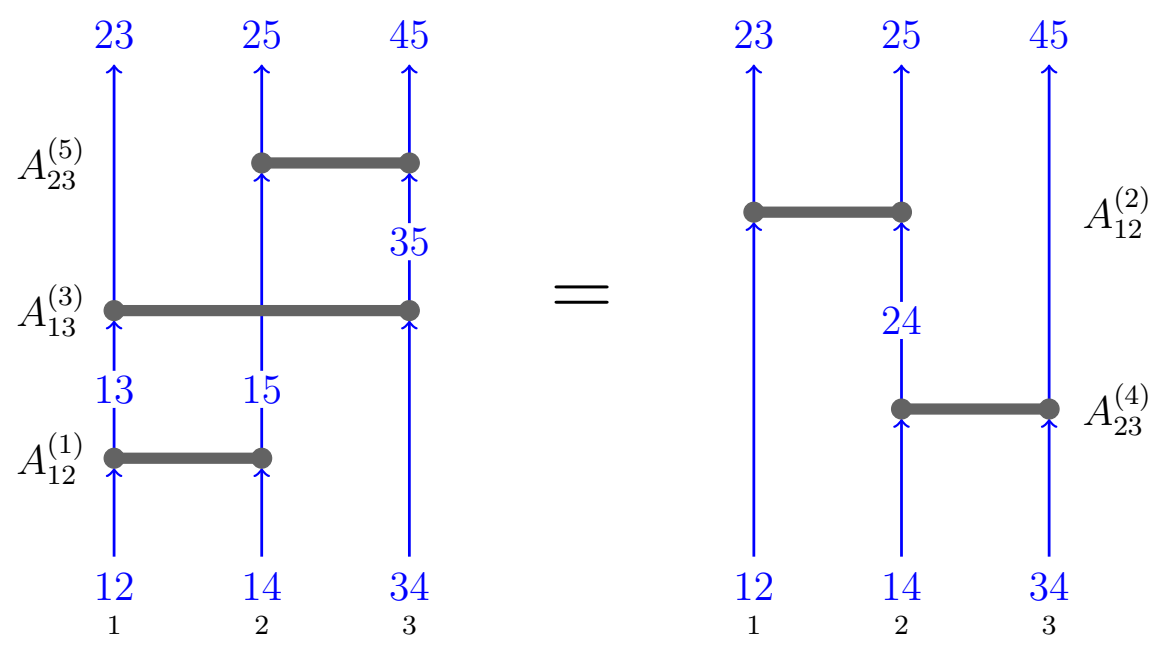

Figure 1: Pentagon equation. Action of 1.h.s. or r.h.s. on row vectors corresponds to going from the bottom to the top in this figure. The left-hand side is to be compared with the blue part of Figure 2

Example 1. Figure 1 shows what happens with index pairs for $n=2$, that is, pentagon equation.

If we take the inverses of both sides of (5), and interchange its l.h.s. and r.h.s., we get equation

$$
B_{\mathcal{B}_{2}}^{(2)} B_{\mathcal{B}_{4}}^{(4)} \cdots B_{\mathcal{B}_{2 n}}^{(2 n)}=B_{\mathcal{B}_{2 n+1}}^{(2 n+1)} B_{\mathcal{B}_{2 n-1}}^{(2 n-1)} \cdots B_{\mathcal{B}_{1}}^{(1)}
$$

where

$$
B^{(q)}=\left(A^{(q)}\right)^{-1} .
$$

The natural indexation of row entries for (12) is obtained from (10) by interchanging the rows in its l.h.s. and r.h.s.:

$$
\left(\begin{array}{llll}
u_{p_{2}, q} & u_{p_{4}, q} & \ldots & u_{p_{2 n}, q}
\end{array}\right) B^{(q)}=\left(\begin{array}{llll}
u_{p_{1}, q} & u_{p_{3}, q} & \ldots & u_{p_{2 n-1}, q}
\end{array}\right) .
$$

As we will see, having solutions to (51) together with solutions to (12) turns out to be important for constructing solutions to $2 n$-simplex equation. We would like to note here that transposed matrices $B^{(q)}=\left(A^{(q)}\right)^{\mathrm{T}}$ also satisfy (12). In this paper, however, we will be working with the case (13).

\section{$2.32 n$-simplex equation}

The direct sum $(2 n)$-simplex has the form

$$
R_{\mathcal{A}_{1}}^{(1)} R_{\mathcal{A}_{2}}^{(2)} \cdots R_{\mathcal{A}_{2 n+1}}^{(2 n+1)}=R_{\mathcal{A}_{2 n+1}}^{(2 n+1)} \cdots R_{\mathcal{A}_{2}}^{(2)} R_{\mathcal{A}_{1}}^{(1)}
$$


Here $R^{(q)}, q=1,2, \ldots, 2 n+1$ are $(2 n) \times(2 n)$-matrices. The equation is defined on a vector space of dimension $\left(\begin{array}{c}2 n+1 \\ 2\end{array}\right)=n(2 n+1)$ consisting of rows which we write as $\left(\begin{array}{llll}u_{1,2} & u_{1,3} & \ldots & u_{2 n, 2 n+1}\end{array}\right)$. The indices here go in lexicographic order, and in a more detailed form they are

$$
\begin{aligned}
& (1,2),(1,3), \ldots,(1,2 n+1), \ldots, \\
& \quad(\boldsymbol{k}, \boldsymbol{k}+\mathbf{1}), \ldots,(\boldsymbol{k}, \mathbf{2 n}+\mathbf{1}), \ldots,(2 n, 2 n+1),
\end{aligned}
$$

where we again highlighted a "typical subsequence" like we did in (7) and (8). In contrast with the $(2 n+1)$-gon equation of Subsection 2.2, the double indexing (16) is the same for the input and output positions where each matrix $R^{(q)}$ acts. Namely, these are the positions where $q$ is met in the double index; we can write it in the following way:

$$
\left(\begin{array}{llll}
u_{p_{1}, q} & u_{p_{2}, q} & \ldots & u_{p_{2 n}, q}
\end{array}\right) R^{(q)}=\left(\begin{array}{llll}
v_{p_{1}, q} & v_{p_{2}, q} & \ldots & v_{p_{2 n}, q}
\end{array}\right),
$$

identifying if needed $\left(p_{i}, q\right)$ with $\left(q, p_{i}\right)$.

Sets $\mathcal{A}_{q}$ consist, correspondingly, of the positions of double indices in (17) in the sequence (16). We denote these positions as follows:

$$
\mathcal{A}_{q}=\left\{a_{q, 1}, \ldots, a_{q, 2 n}\right\}
$$

similarly to (6) . Concerning explicit formulas for $a_{q, i}$, see Proposition 2 below.

\subsection{Positions where matrices act nontrivially}

Proposition 2. Positions where matrices act nontrivially in the 2 -simplex equation are given by

$$
a_{k, j}=\left\{\begin{array}{cc}
\frac{(4 n-k)(k-1)}{2}+j & \text { for } j \geq k, \\
a_{j, k-1} & \text { for } j<k .
\end{array}\right.
$$

Formula (19) first appeared, without proof, in [4, p. 16].

Proof. Case $j \geq k$. For such $j$, as one can readily see from (16), $a_{k, j}=r_{k}+j$, where

$$
\begin{aligned}
& r_{1}=0, \quad r_{2}=2 n-1, \quad r_{3}=r_{2}+(2 n-2), \quad \ldots, \\
& r_{k}=(2 n-1)+(2 n-2)+\cdots+(2 n-k+1)=\frac{(4 n-k)(k-1)}{2} .
\end{aligned}
$$

Case $j<k$. Indeed, in this case pair $j, k$ in (16) corresponds to both $a_{k, j}$ and $a_{j, k-1}$; see also Example 2 below. 
Example 2. To illustrate Proposition 2, take $n=2$, that is, the 4 -simplex equation. Here are the rows consisting of index pairs corresponding to $\mathcal{A}_{k}$; those pairs where $j<k$ are highlighted with color:

\begin{tabular}{c|cccc} 
& $j=1$ & $j=2$ & $j=3$ & $j=4$ \\
\hline$k=1$ & 12 & 13 & 14 & 15 \\
$k=2$ & 12 & 23 & 24 & 25 \\
$k=3$ & 13 & 23 & 34 & 35 \\
$k=4$ & 14 & 24 & 34 & 45 \\
$k=5$ & 15 & 25 & 35 & 45
\end{tabular}

We now introduce $\boldsymbol{n}$-simplex equation, which turns out to be interesting because of its connection with the $(\mathbf{2 n}+\mathbf{1})$-gon. All the content of the previous Subsection 2.3 and Proposition 2 remain perfectly valid if we change everywhere $2 n$ to just $n$. To distinguish analogues of sets (18) from the " $2 n$ " case, we add a bar to letters $\mathcal{A}$ and $a$ in the " $n$ " case:

$$
\begin{aligned}
\overline{\mathcal{A}}_{k} & =\left\{\bar{a}_{k, 1}, \ldots, \bar{a}_{k, n}\right\}, \\
\bar{a}_{k, j} & =\left\{\begin{array}{cc}
\frac{(2 n-k)(k-1)}{2}+j & \text { for } j \geq k, \\
\bar{a}_{j, k-1} & \text { for } j<k .
\end{array}\right.
\end{aligned}
$$

Proposition 3. Positions where matrices act nontrivially in the $(2 n+1)$-gon equation are given by

$$
\begin{aligned}
& \mathcal{B}_{2 k-1}=\overline{\mathcal{A}}_{k}, \\
& \mathcal{B}_{2 k}=\overline{\mathcal{A}}_{k}+b_{k}, \quad \text { with } \quad b_{k}=\left(\beta_{k, 1}, \ldots, \beta_{k, n}\right), \quad \beta_{k, j}= \begin{cases}0, & j \geq k, \\
1, & j<k .\end{cases}
\end{aligned}
$$

Proof. It follows from the analysis made in the proof of Proposition 1 that $\mathcal{B}_{2 k-1}$ can be described in terms of a fixed - not changing with the action of any $A^{(2 k-1)}$ - sequence of double indices. Namely, take, for each pair, the first member from the corresponding pair in (7), while the second from the corresponding pair in (8). We obtain the following sequence involving only odd numbers (where we highlight a "typical subsequence" like we did earlier):

$$
\begin{gathered}
(1,3),(1,5), \ldots,(1,2 n+1), \ldots, \\
(\mathbf{2 l}-\mathbf{1}, \mathbf{2 l}+\mathbf{1}),(\mathbf{2 l}-\mathbf{1}, \mathbf{2 l}+\mathbf{3}), \ldots,(\mathbf{2 l}-\mathbf{1}, \mathbf{2 n}+\mathbf{1}), \\
\ldots,(2 n-1,2 n+1),
\end{gathered}
$$

and $\mathcal{B}_{2 k-1}$ consists, as can be readily seen, exactly of such positions where $2 k-1$ is a member of the corresponding pair.

Compare now (24) with the analogue of (16) for the $n$-simplex:

$$
\begin{aligned}
& (1,2),(1,3), \ldots,(1, n+1), \ldots, \\
& \quad(\boldsymbol{l}, \boldsymbol{l}+\mathbf{1}),(\boldsymbol{l}, \boldsymbol{l}+\mathbf{2}), \ldots,(\boldsymbol{l}, \boldsymbol{n}+\mathbf{1}), \ldots,(n, n+1) .
\end{aligned}
$$


Equality (22) is now clear from the fact that for any number $m$ entering in (25), there is $(2 m-1)$ at the corresponding place in (24).

Similarly, $\mathcal{B}_{2 k}$ can be described as consisting of such positions where $2 k$ is a member of the pair in the following fixed - not changing with the action of any $A^{(2 k)}$ _ sequence: take, for each pair, the first member from the corresponding pair in (8), while the second from the corresponding pair in (7) (so, we write a pair of coinciding numbers if these two coincide). We obtain the following sequence involving only even numbers (we highlight again a "typical subsequence"):

$$
\begin{aligned}
(2,2),(2,4), \ldots,(2,2 n), \ldots \\
(\mathbf{2 l}, \mathbf{2 l}),(\mathbf{2 l}, \mathbf{2 l}+\mathbf{2}), \ldots,(\mathbf{2 l}, \mathbf{2 n}), \ldots,(2 n, 2 n),
\end{aligned}
$$

Comparing (26) with (25), one arrives, after a simple analysis, at equality (23).

\subsection{Three-color decomposition}

As we have already said, each matrix $R^{(q)}$ from the $2 n$-simplex equation (15) acts nontrivially on its own $2 n$-row, see (17). Consider now one special type of $R$-matrices, for which the even entries of the output row (r.h.s. of (17)) depend only on odd entries of the input row (in the l.h.s. of (17)), and vice versa. That is, we can represent in this case the dependence (17) as

$$
\begin{gathered}
\left(\begin{array}{llll}
u_{p_{1}, q} & u_{p_{3}, q} & \ldots & u_{p_{2 n-1}, q}
\end{array}\right) A^{(q)}=\left(\begin{array}{lllll}
v_{p_{2}, q} & v_{p_{4}, q} & \ldots & v_{p_{2 n}, q}
\end{array}\right), \\
\left(\begin{array}{lllll}
u_{p_{2}, q} & u_{p_{4}, q} & \ldots & u_{p_{2 n}, q}
\end{array}\right) B^{(q)}=\left(\begin{array}{llll}
v_{p_{1}, q} & v_{p_{3}, q} & \ldots & v_{p_{2 n-1}, q}
\end{array}\right)
\end{gathered}
$$

where $A^{(q)}$ and $B^{(q)}$ are the corresponding submatrices of $R^{(q)}$ (and all entries of $R^{(q)}$ outside $A^{(q)}$ and $B^{(q)}$ are zero). Of course we called them $A^{(q)}$ and $B^{(q)}$ intentionally, because the indexation in (27) coincides exactly with (10) and (14).

Such matrices can be said to admit a factorization in $A^{(q)}, B^{(q)}$, and permutation matrices $P_{i, j}$, because (27) is equivalent to

$$
\begin{aligned}
R^{(q)} & =A_{1,3, \ldots, 2 n-1}^{(q)} B_{2,4, \ldots, 2 n}^{(q)} P_{1,2} P_{3,4} \cdots P_{2 n-1,2 n} \\
& =A_{1,3, \ldots, 2 n-1}^{(q)} P_{1,2} P_{3,4} \cdots P_{2 n-1,2 n} B_{1,3, \ldots, 2 n-1}^{(q)}, \quad P=\left(\begin{array}{cc}
0 & 1 \\
1 & 0
\end{array}\right) .
\end{aligned}
$$

Subscripts in (28) correspond to rows and columns of $R^{(q)}$ viewed as a $(2 n \times 2 n)$ matrix.

We now assign colors - blue, red and green - to each entry in the initial $n(2 n+1)$-row (on which both l.h.s. and r.h.s. of (15) act), as well as in all the middle rows and the final row, according to the following rules. In the initial row, the blue color is assigned to entries in positions, or simply "to positions", (77); the red color is assigned to positions (8); and the green color to all the remaining 
positions. Then, taking either l.h.s. or r.h.s., we propagate the colors through each $R^{(q)}$ in such way that the entries in the r.h.s. of (27) acquire the same color as those in the corresponding l.h.s. of (27). It is important to note that this process goes ahead without obstacles or contradictions, and this is because the "blue sector" occupies exactly the places indexed in the same way as we did for the $(2 n+1)$-gon equation (5), while the "red sector" - indexed in the same way as for (12); the rest of the places remain for the green sector.

We now sum it up as the following proposition.

Proposition 4. For $R$-matrices admitting factorization of type (28), the $2 n$ simplex equation can be decomposed into three independent parts: "blue", "red" and "green", the blue part being the $(2 n+1)$-gon equation (5), while the red part-the inverse $(2 n+1)$-gon (12) equation.

Thus, blue part involves only matrices $A^{(q)}$, red part-only $B^{(q)}$, and the green part, as one can see for instance from Example 3 below, is a mixed equation for both $A^{(q)}$ and $B^{(q)}$.

Some important properties of the green sector deserve a separate proposition.

\section{Proposition 5.}

(i) The initial and final positions in the green sector are the same, namely having both indices either odd or even:

$$
\begin{aligned}
& (2 j, 2 k), \quad 1 \leq j<k \leq n \\
& \text { or }(2 j+1,2 k+1), \quad 0 \leq j<k \leq n .
\end{aligned}
$$

(ii) The inner (not initial or final) positions in the green sector all have one index odd and the other even, that is, belong to one of the sequences (7) and (8). Moreover, any position in (71) and (8) is met exactly one time in the green sector.

Proof. (i) This follows from the fact that positions (7) are initial and positions (8) are final for the blue sector, while positions (8) are initial and positions (7) are final for the red sector; what remains is "green".

(ii) (a) First, we note that there are exactly two $R$-matrices changing the entry at position $(j, k)$, namely $R^{(j)}$ and $R^{(k)}$. So, there is one "initial" row entry at a given position, one "inner", and one "final".

(b) It follows that, for a chosen $R^{(q)}$ and a position $(q, l)(=(l, q))$, either the input row entry $u_{q, l}$ is initial or the output row entry $v_{q, l}$ is final. Indeed, the first case happens if $R^{(q)}$ acts before $R^{(l)}$, while the second case happens if $R^{(q)}$ acts after $R^{(l)}$.

(c) It follows then from (b) that there is no matrix $R^{(q)}$ belonging wholly to a single color sector. Indeed, it can be easily seen from (29) that, for a given $q$, some of positions $(q, l)(=(l, q))$ are green if considered as either initial or final, while some other are not. 
(d) Thus, the color at each given position is changed with the action of any $R$-matrix. This together with the preceding analysis shows that there are exactly four possibilities for the initial-inner-final colors: blue-green-red, red-green-blue, green-blue-green and greenred-green. Hence, item (ii) follows.

It follows from Proposition 5 (i) that any side of (15) is a direct sum of two submatrices, acting one in the green sector, while the other in the blue and red

sectors. Moreover, the latter submatrix, due to the fact that $B^{(q)}=\left(A^{(q)}\right)^{-1}$ in our construction, has the obvious block structure

$$
\left(\begin{array}{cc}
0 & K^{-1} \\
K & 0
\end{array}\right)
$$

Example 3. For $n=2$, we have a 4 -simplex equation, a heptagon (7-gon) equation, and the following coloring of (16) (where we write simply "12" instead of "( $(1,2)$ ", etc.):

$$
(12,13,14,15,23,24,25,34,35,45) \text {. }
$$

The 4-simplex equation reads

$$
R_{1234}^{(1)} R_{1567}^{(2)} R_{2589}^{(3)} R_{3680}^{(4)} R_{4790}^{(5)}=R_{4790}^{(5)} R_{3680}^{(4)} R_{2589}^{(3)} R_{1567}^{(2)} R_{1234}^{(1)},
$$

and can be re-written, using (28), as

$$
\begin{aligned}
\left(A_{13}^{(1)} A_{18}^{(3)} A_{38}^{(5)}\right)\left(B_{24}^{(1)} A_{26}^{(2)} B_{29}^{(3)} A_{49}^{(4)} B_{69}^{(5)}\right)\left(B_{57}^{(2)} B_{70}^{(4)}\right) & \\
= & \left(A_{38}^{(4)} A_{13}^{(2)}\right)\left(A_{49}^{(5)} B_{69}^{(4)} A_{29}^{(3)} B_{24}^{(2)} A_{26}^{(1)}\right)\left(B_{70}^{(5)} B_{50}^{(3)} B_{57}^{(1)}\right),
\end{aligned}
$$

where the equality holds also for each separate color.

The left-hand side of either (31) or (32) can be visualized as in Figure 2 ,

\subsection{Reduction to $(2 n-1)$-simplex}

If we have a solution of direct-sum $2 n$-simplex equation (15), we can, under some mild conditions, apply to it a reduction procedure and obtain a solution of the "lower" equation, namely $(2 n-1)$-simplex. Here we describe such a procedurean analogue of taking "partial trace" in the quantum case - and, specifically, what it gives for $R$-operators (28).

Each $R$-matrix in (15) imposes $2 n$ linear conditions on $4 n$ variables - $2 n$ input and $2 n$ output. In this Subsection, we would like to denote its input and output rows as follows:

$$
\left(\begin{array}{llll}
u_{1}^{(q)} & u_{2}^{(q)} & \ldots & u_{2 n}^{(q)}
\end{array}\right) R^{(q)}=\left(\begin{array}{llll}
v_{1}^{(q)} & v_{2}^{(q)} & \ldots & v_{2 n}^{(q)}
\end{array}\right) .
$$




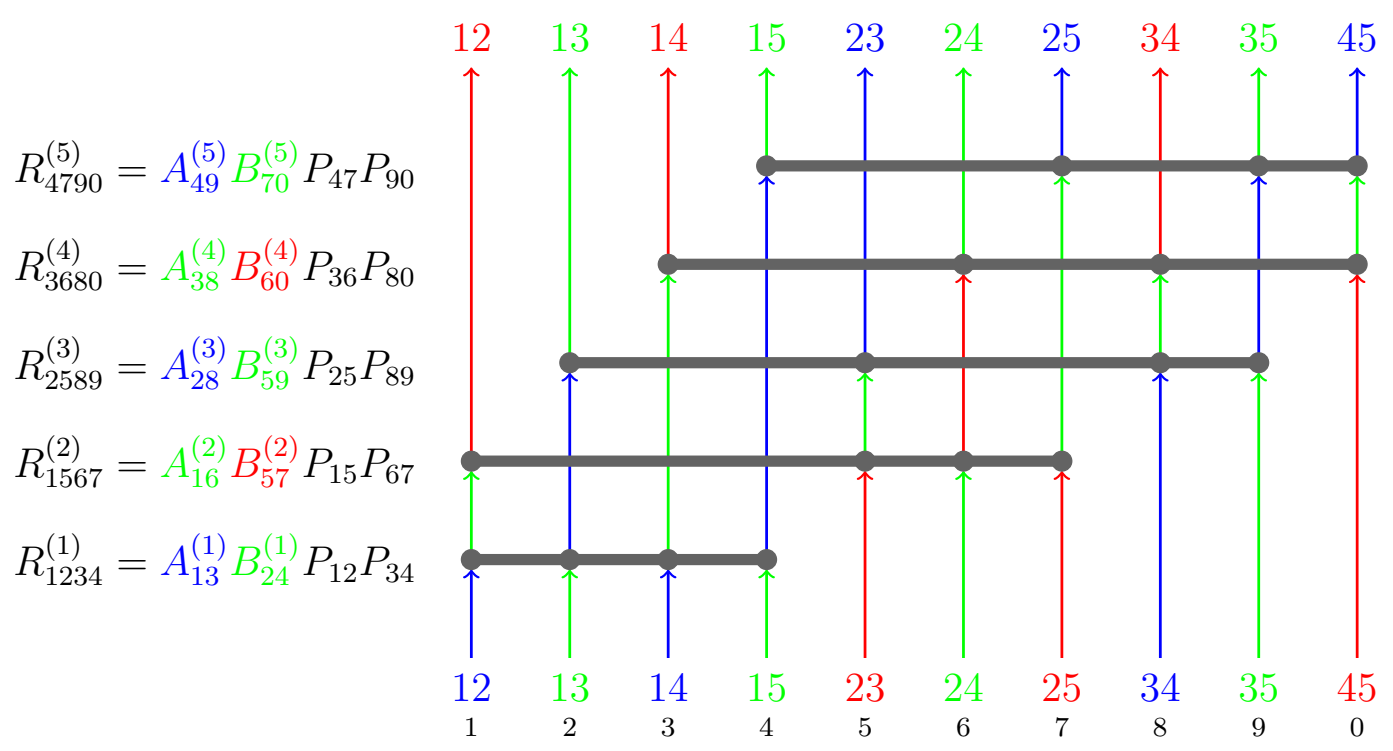

Figure 2: The left-hand side of (31) corresponds to going from the bottom to the top of this picture

We take then a number $\lambda \in F$ and impose the following additional condition:

$$
u_{2 n}^{(q)}=\lambda v_{2 n}^{(q)}
$$

and do it for all $R$-matrices except the last, that is, for $R_{\mathcal{A}_{1}}^{(1)}, \ldots, R_{\mathcal{A}_{2 n}}^{(2 n)}$. We assume that, for each of these $R$-matrices, (33) together with (34) determine also $u_{2 n}^{(q)}$ from the rest of input variables (which is usually the case). Hence, in particular, variables $v_{1}^{(q)}, v_{2}^{(q)}, \ldots, v_{2 n-1}^{(q)}$ are determined given $u_{1}^{(q)}, u_{2}^{(q)}, \ldots, u_{2 n-1}^{(q)}$, and we denote $Z^{(q)}$ the matrix giving these dependencies:

$$
\left(\begin{array}{llll}
u_{1}^{(q)} & u_{2}^{(q)} & \ldots & u_{2 n-1}^{(q)}
\end{array}\right) Z^{(q)}=\left(\begin{array}{llll}
v_{1}^{(q)} & v_{2}^{(q)} & \ldots & v_{2 n-1}^{(q)}
\end{array}\right) .
$$

Proposition 6. Matrices $Z^{(q)}$ satisfy the $(2 n-1)$-simplex equation

$$
Z^{(1)} Z^{(2)} \ldots Z^{(2 n)}=Z^{(2 n)} \ldots Z^{(2)} Z^{(1)}
$$

In (36), we did not write out the positions where matrices $Z^{(q)}$ act; these are defined in complete analogy with Subsection 2.3.

Proof. First, write a special input row for the whole $2 n$-simplex (15), choosing last entries $u_{2 n}^{(q)}$ obeying (34) for all $R$-matrices in the l.h.s. except $R^{(2 n+1)}$ : first for $R^{(1)}$, then for $R^{(2)}, \ldots$, finally for $R^{(2 n)}$. No conditions are imposed on the other input entries. 
As $\lambda$ is the same for all the mentioned $R^{(q)}$, and $R^{(2 n+1)}$ acts exactly on the last positions of all other matrices, the input rows for $R^{(2 n+1)}$ in the l.h.s. and r.h.s. of (15) are proportional: in the r.h.s., they are $\lambda$ times those in the l.h.s. Hence, the same holds also for the output rows, and we can describe what happens at such a position under action of two matrices as follows (denoting $w_{2 n}^{(q)}$ the final value at this position):

$$
\begin{array}{ll}
\text { l.h.s.: } \quad u_{2 n}^{(q)}=\lambda v_{2 n}^{(q)} \stackrel{R^{(q)}}{\longmapsto} v_{2 n}^{(q)} \stackrel{{ }^{(2 n+1)}}{\longmapsto} w_{2 n}^{(q)}, \\
\text { r.h.s.: } \quad u_{2 n}^{(q)}=\lambda v_{2 n}^{(q)} \stackrel{R^{(2 n+1)}}{\longmapsto} \lambda w_{2 n}^{(q)} \stackrel{R^{(q)}}{\longmapsto} w_{2 n}^{(q)} .
\end{array}
$$

Indeed, the values around the first arrow in (38) must be proportional to those around the second arrow in (37). But then we see from the second arrow in (38) that the same condition as (34) holds for $R^{(q)}$ also in the r.h.s., hence, its reduction $Z^{(q)}$ is the same matrix in both sides, and (36) holds. by

Explicit expressions for matrices $Z^{(q)}$ in the case of $R^{(q)}$ as in (28) are given

$$
\begin{array}{r}
Z^{(q)}=A_{1,3, \ldots, 2 n-1}^{(q)} P_{12} P_{34} \cdots P_{2 n-3,2 n-2} \Lambda_{2 n-1} B_{1,3, \ldots, 2 n-1}^{(q)}, \\
\Lambda_{2 n-1}=\left(\begin{array}{cc}
\mathbb{1}_{2 n-2} & 0 \\
0 & \lambda
\end{array}\right),
\end{array}
$$

as a small exercise shows. Formula (39) can be treated as a generalization of (the direct-sum analogue of) the tetrahedron equation solution in [11, (1.8)].

Note that $\lambda=0$ can also be taken in (34), and the obtained solutions in this case are

$$
Z^{(q)}=S^{\mathrm{T}} R^{(q)} S, \quad S=\left(\begin{array}{c}
\mathbb{1}_{2 n-1} \\
0
\end{array}\right), \quad q=1,2, \ldots, 2 n-1,
$$

that is, $Z^{(q)}$ is simply the submatrix of $R^{(q)}$ obtained by deleting its last row and column.

Finally, we note that the described kind of reduction can be applied not one but several times: from $(2 n-1)$-simplex to $(2 n-2)$-simplex and so on. The solutions of simplex equations thus obtained deserve to be the subject of a separate research. 


\section{Construction of solutions}

\subsection{Multivectors $\phi$ and $\psi$ on which the construction is based}

Let $F$ be a field, $F^{2 n+1}$ the space of $(2 n+1)$-rows, $\mathrm{e}_{1}, \ldots, \mathrm{e}_{2 n+1}$ the standard basis in $F^{2 n+1}$, and $\mathrm{e}^{1}, \ldots, \mathrm{e}^{2 n+1}$ the dual basis in the dual space. Let

$$
\mathcal{M}=\left(\begin{array}{cccc}
\alpha_{1,1} & \alpha_{1,2} & \cdots & \alpha_{1,2 n+1} \\
\alpha_{2,1} & \alpha_{2,2} & \cdots & \alpha_{2,2 n+1} \\
\vdots & \vdots & \ddots & \vdots \\
\alpha_{n+1,1} & \alpha_{n+1,2} & \cdots & \alpha_{n+1,2 n+1}
\end{array}\right)
$$

be a matrix defining an element $L$ of the Grassmannian $\operatorname{Gr}(n+1,2 n+1)$, that is, a matrix of the full rank $n+1$ whose rows, which can also be written as

$$
v_{i}=\alpha_{i, 1} \mathrm{e}_{1}+\cdots+\alpha_{i, 2 n+1} \mathrm{e}_{2 n+1},
$$

span an $(n+1)$-dimensional plane $L \subset F^{2 n+1}$.

Below, we will be working with multivectors - elements of the exterior algebra $\bigwedge F^{2 n+1}$ over $F^{2 n+1}$. First, we introduce an $(n+1)$-vector - the exterior product of all $v_{i}$ :

$$
w=v_{1} \wedge v_{2} \wedge \cdots \wedge v_{n+1}=\sum_{k_{1}<\cdots<k_{n+1}} p_{k_{1}, \ldots, k_{n+1}} \mathrm{e}_{k_{1}} \wedge \cdots \wedge \mathrm{e}_{k_{n+1}},
$$

where $p_{k_{1}, \ldots, k_{n+1}}$ are determinants made of $k_{1}$-th, $\ldots, k_{n+1}$-th columns of $\mathcal{M}$, called also Plücker coordinates of the Grassmannian $\operatorname{Gr}(n+1,2 n+1)$.

We will also need the convolution operation $\lrcorner$, see, for instance, [19, page 42]. In our context, this will be essentially the same as the left Grassmann derivative [2, Eq. (2.1.2)]: for instance, $\phi^{i, j}$ below in formula (41) can be written also as $\frac{\partial}{\partial \mathrm{e}_{j}} \frac{\partial}{\partial \mathrm{e}_{i}} w$.

Assumption 1. Below, we assume that all Plücker coordinates are nonzero.

Under Assumption 1, field $F$ must not be too small, as the following example shows. A deeper analysis using the "irrelevance of algebraic inequalities" principle shows that Assumption 1 can be partially relaxed, because what we ultimately need is that there must be no division by zero in formulas (44) and (45) below. This may be important when working with finite fields; we leave these questions for future research.

Example 4. For $n=2$, the smallest field allowing to construct matrix $\mathcal{M}$ (40) obeying Assumption 1 is the Galois field $\mathbb{F}_{4}$ of four elements. For instance,

$$
\mathcal{M}=\left(\begin{array}{llllc}
1 & 0 & 0 & 1 & 1 \\
0 & 1 & 0 & 1 & \zeta \\
0 & 0 & 1 & 1 & \zeta^{2}
\end{array}\right)
$$


where $\zeta \in \mathbb{F}_{4}$ is not equal to either 0 or 1 .

We define $(n-1)$-vectors

$$
\left.\phi^{i, j}=\left(\mathrm{e}^{j} \wedge \mathrm{e}^{i}\right)\right\lrcorner w=\sum_{k_{1}<\cdots<k_{n-1}} p_{i, j, k_{1}, \ldots, k_{n-1}} \mathrm{e}_{k_{1}} \wedge \cdots \wedge \mathrm{e}_{k_{n-1}},
$$

and $(n+3)$-vectors

$$
\psi_{i, j}=\mathrm{e}_{i} \wedge \mathrm{e}_{j} \wedge w=\sum_{k_{1}<\cdots<k_{n+1}} p_{k_{1}, \ldots, k_{n+1}} \mathrm{e}_{i} \wedge \mathrm{e}_{j} \wedge \mathrm{e}_{k_{1}} \wedge \cdots \wedge \mathrm{e}_{k_{n+1}} .
$$

Both objects $\phi^{i, j}$ and $\psi_{i, j}$ are antisymmetric in their indices.

Example 5. For $n=3$ we have a 2-vector

$$
\begin{array}{r}
\phi^{1,2}=\sum_{1 \leq k<l \leq 7} p_{1,2, k, l} \mathrm{e}_{k} \wedge \mathrm{e}_{l}=-p_{1,2,3,4} \mathrm{e}_{3} \wedge \mathrm{e}_{4}-p_{1,2,3,5} \mathrm{e}_{3} \wedge \mathrm{e}_{5}-p_{1,2,3,6} \mathrm{e}_{3} \wedge \mathrm{e}_{6} \\
-p_{1,2,3,7} \mathrm{e}_{3} \wedge \mathrm{e}_{7}-p_{1,2,4,5} \mathrm{e}_{4} \wedge \mathrm{e}_{5}-p_{1,2,4,6} \mathrm{e}_{4} \wedge \mathrm{e}_{6}-p_{1,2,4,7} \mathrm{e}_{4} \wedge \mathrm{e}_{7} \\
-p_{1,2,5,6} \mathrm{e}_{5} \wedge \mathrm{e}_{6}-p_{1,2,5,7} \mathrm{e}_{5} \wedge \mathrm{e}_{7}-p_{1,2,6,7} \mathrm{e}_{6} \wedge \mathrm{e}_{7}
\end{array}
$$

and a 6 -vector

$$
\begin{array}{r}
\psi_{2,3}=p_{1,4,5,6} e_{1} \wedge e_{2} \wedge e_{3} \wedge e_{4} \wedge e_{5} \wedge e_{6}+p_{1,4,5,7} e_{1} \wedge e_{2} \wedge e_{3} \wedge e_{4} \wedge e_{5} \wedge e_{7} \\
+p_{1,4,6,7} e_{1} \wedge e_{2} \wedge e_{3} \wedge e_{4} \wedge e_{6} \wedge e_{7}+p_{1,5,6,7} e_{1} \wedge e_{2} \wedge e_{3} \wedge e_{5} \wedge e_{6} \wedge e_{7} \\
+p_{4,5,6,7} e_{2} \wedge e_{3} \wedge e_{4} \wedge e_{5} \wedge e_{6} \wedge e_{7}
\end{array}
$$

\subsection{Dimensions of linear spaces spanned by multivectors}

Proposition 7. If we fix index $j$ in $\phi^{i, j}$, while letting $i$ take any $n$ different values $i \neq j$, then the $n$ resulting multivectors are linearly independent.

Proof. Denote the chosen values of $i$ as $i_{1}<\cdots<i_{n}$. Suppose there is a vanishing linear combination

$$
\sum_{l=1}^{n} \lambda_{l} \phi^{i_{l}, j}=0
$$

of the mentioned multivectors. We are going to show that all its coefficients $\lambda_{l}$ are zero.

Fix $l$, and consider, for all $i=i_{1}, \ldots, i_{n}$, the corresponding coefficients of $\mathrm{e}_{i_{1}} \wedge \cdots \wedge \hat{e}_{i_{l}} \wedge \cdots \wedge \mathrm{e}_{i_{n}}$ in the r.h.s. of (41). Clearly, they all vanish except the $l$-th one which is $\lambda_{l} p_{i_{l}, j, i_{1}, \ldots, \hat{i}_{l}, \ldots, i_{n}}$. Combined with Assumption 1, this immediately gives $\lambda_{l}=0$.

Proposition 8. The linear space spanned by multivectors $\phi^{i, j}$ with a fixed $j$ is exactly $n$-dimensional. 
Proof. There are only $(n+1)$ linearly independent among expressions $\left.\mathrm{e}^{i}\right\lrcorner w$ for all $i$. Further action of $\mathrm{e}^{j}$, that is, taking $\left.\left(\mathrm{e}^{j} \wedge \mathrm{e}^{i}\right)\right\lrcorner w$ as in (41), kills one of these, so, $n$ linearly independent expressions remain.

Proposition 9. The multivectors $\phi^{i, j}$ on which either l.h.s. or r.h.s. of the $(2 n+1)$-gon relation acts, are linearly independent, that is, span an $\frac{n(n+1)}{2}$ dimensional linear space.

Proof. We have to show that

$$
\sum_{j=1}^{n} \sum_{k=j}^{n} \lambda_{2 j-1,2 k} \phi^{2 j-1,2 k}=0
$$

implies $\lambda_{2 j-1,2 k}=0$ for all $j, k$ entering in the above double sum.

We note first that

$$
\mathrm{e}_{1} \wedge \mathrm{e}_{3} \wedge \cdots \wedge \mathrm{e}_{2 n-5} \wedge \mathrm{e}_{2 n-3} p_{1,3, \ldots, 2 n-3,2 n-1,2 n}
$$

appears only in $\phi^{2 n-1,2 n}$ hence $\lambda_{2 n-1,2 n}=0$. After that we note next that

$$
\mathrm{e}_{1} \wedge \mathrm{e}_{3} \wedge \cdots \wedge \mathrm{e}_{2 n-5} \wedge \mathrm{e}_{2 n-2} p_{1,3, \ldots, 2 n-3,2 n-2,2 n}
$$

appears only in $\phi^{2 n-3,2 n}$ hence $\lambda_{2 n-3,2 n}=0$. Continuing in this way suppose we have proved that $\lambda_{2 n-2 j-1,2 n}=0$ for $j=1,2, \ldots, k-1$, then the term

$$
\mathrm{e}_{1} \wedge \mathrm{e}_{3} \wedge \cdots \wedge \mathrm{e}_{2 n-2 k-1} \wedge \mathrm{e}_{2 n-2 k} \wedge \cdots \wedge \mathrm{e}_{2 n-2} p_{1,3, \ldots, 2 n-2 k-1,2 n-2 k, \ldots, 2 n-2,2 n}
$$

appears only in $\phi^{2 n-2 k-1,2 n}$ hence $\lambda_{2 n-2 k-1,2 n}=0$. By induction $\lambda_{2 k-1,2 n}=0$ for $k=1,2, \ldots, n$.

Next we look for the term

$$
\mathrm{e}_{1} \wedge \mathrm{e}_{3} \wedge \cdots \wedge \mathrm{e}_{2 n-3} \wedge \mathrm{e}_{2 n-2} \wedge \mathrm{e}_{2 n} p_{1,3, \ldots, 2 n-3,2 n-2,2 n}
$$

from which $\lambda_{2 n-3,2 n-2}=0$ follows, etc.

Now a proposition about $\phi$ 's and $\psi$ 's in the green sector of the $2 n$-simplex relation. The green sector acts on those pairs $i, j$ where either both $i$ and $j$ are even, or both $i$ and $j$ are odd. It is enough for us to consider just the odd $i$ and $j$ for $\phi$ 's, and just the even $i$ and $j$ for $\psi$ 's.

\section{Proposition 10.}

(i) The $\frac{n(n+1)}{2}$ multivectors $\phi_{i, j}$ for all odd $i$ and $j$ are linearly independent.

(ii) The $\frac{n(n-1)}{2}$ multivectors $\psi_{i, j}$ for all even $i$ and $j$ are linearly independent. 
We will see when proving Theorem 2 that actually the space spanned by all $\phi_{i, j}$ in the green sector is exactly $\frac{n(n+1)}{2}$-dimensional, while the space spanned by all $\psi_{i, j}$ in the green sector is exactly $\frac{n(n-1)}{2}$-dimensional.

Proof. The proofs of (i) and (ii) are almost identical, only with some obvious changes, so it is enough to write out here only the proof of (ii). Suppose, like in the proofs of Propositions 7 and 9, that there is a dependence

$$
\sum_{\substack{i, j \text { even } \\ i<j}} \lambda_{i, j} \psi_{i, j}=0
$$

choose any $i=i_{0}$ and $j=j_{0}$, and consider the coefficients of

$$
\mathrm{e}_{i_{0}} \wedge \mathrm{e}_{j_{0}} \wedge \mathrm{e}_{1} \wedge \mathrm{e}_{3} \wedge \cdots \wedge \mathrm{e}_{2 n+1}
$$

using now of course the r.h.s. of (42) for $\psi_{i, j}$. The same argument as in the mentioned proofs shows that $\lambda_{i_{0}, j_{0}}=0$.

\subsection{Matrices $A^{(q)}$ and $B^{(q)}$ and their relations to $\phi$ and $\psi$}

We are going to define matrices $A^{(q)}$ and $B^{(q)}=\left(A^{(q)}\right)^{-1}$ that will give us solutions to both $(2 n+1)$-gon and $2 n$-simplex equations. Technically, we prefer to give here first explicit expressions (44) and (45) for their matrix entries, and then prove their key properties in Proposition 11. It must be noted, however, that, conceptually, the reason for existence of $A^{(q)}$ and $B^{(q)}$ satisfying (46) and (48) below lies in the dimension count given in Propositions 7 and 8 . That is, the mentioned propositions guarantee that formulas (46) and (48) below can be used as correct definitions of $A^{(q)}$ and $B^{(q)}$ respectively (and as for Proposition 9, it will be used for proving the $(2 n+1)$-gon relation, see Theorem 1).

For $q \in[2 n+1]=\{1,2, \ldots, 2 n+1\}$ we denote

$$
L_{q}=[2 n+1] \backslash\{q\}=\left\{a_{1}, a_{2}, \ldots, a_{2 n}\right\}
$$

(slightly changing notations with respect to (9); remember also Convention 1) and define the following two families of $(n \times n)$-matrices:

$$
\begin{array}{r}
\left(A^{(q)}\right)_{i}^{j}=(-1)^{i} \frac{p_{a_{2 j}, a_{1}, a_{3}, \ldots, \hat{a}_{2 i-1}, \ldots, a_{2 n-1}, q}}{p_{a_{1}, a_{3}, \ldots, a_{2 n-1}, q}}, \\
\left(B^{(q)}\right)_{i}^{j}=(-1)^{i} \frac{p_{a_{2 j-1}, a_{2}, a_{4}, \ldots, \hat{a}_{2 i}, \ldots, a_{2 n}, q}}{p_{a_{2}, a_{4}, \ldots, a_{2 n}, q}}, \\
q=1,2, \ldots, 2 n+1 .
\end{array}
$$

Here index $i$ numbers the rows, while $j$ - the columns of an $n \times n$ matrix. 
Proposition 11. The following equalities hold:

$$
\begin{gathered}
\sum_{i=1}^{n} \phi^{a_{2 i-1}, q}\left(A^{(q)}\right)_{i}^{j}=-\phi^{a_{2 j}, q} \\
\sum_{j=1}^{n}\left(A^{(q)}\right)_{i}^{j} \psi_{a_{2 j}, q}=\psi_{a_{2 i-1, q}} \\
\sum_{i=1}^{n} \phi^{a_{2 i}, q}\left(B^{(q)}\right)_{i}^{j}=-\phi^{a_{2 j-1, q}}, \\
\sum_{j=1}^{n}\left(B^{(q)}\right)_{i}^{j} \psi_{a_{2 j-1}, q}=\psi_{a_{2 i, q}}
\end{gathered}
$$

Note that $A^{(q)}$ and $B^{(q)}$ act on the rows of $\phi$ 's, but on the columns of $\psi$ 's. We have thus extended such formulas as (27) from just scalar row entries to entries taking values in multidimensional linear spaces (and introduced similar column entries). Note also that (13) follows from (46) and (48), because the $\phi$ 's in either side of these formulas span an $n$-dimensional space and thus (46) and (48) unambiguously determine $A^{(q)}$ and $B^{(q)}$, respectively.

Proof. Equality (46) follows from the Plücker relations

$$
\sum_{i=1}^{n}(-1)^{i} p_{a_{2 i}, q, b_{1}, \ldots, b_{n-1}} p_{a_{2 j-1}, a_{2}, \ldots, \hat{a}_{2 i}, \ldots, a_{2 n}, q}+p_{a_{2 j-1}, q, b_{1}, \ldots, b_{n-1}} p_{a_{2}, \ldots, a_{2 n}, q}=0,
$$

while (47) again from the Plücker relations in the form

$$
\left.\left(\left(\mathrm{e}^{a_{1}} \wedge \mathrm{e}^{a_{3}} \wedge \cdots \wedge \hat{\mathrm{e}}^{a_{2 i-1}} \wedge \cdots \wedge \mathrm{e}^{a_{2 n-1}} \wedge \mathrm{e}^{q}\right)\right\lrcorner w\right) \wedge w=0 .
$$

Equalities (48) and (49) are proved similarly.

Note that

$$
\begin{array}{r}
\sum_{j=1}^{n} p_{a_{2 j}, a_{1}, \ldots, \hat{a}_{2 i-1}, \ldots, a_{2 n-1}, \mathrm{q}} \mathrm{e}_{a_{2 j}}-(-1)^{i} p_{a_{1}, a_{3}, \ldots, a_{2 n-1}, q} \mathrm{e}_{a_{2 i-1}} \\
\left.=\left(\mathrm{e}^{a_{1}} \wedge \mathrm{e}^{a_{3}} \wedge \cdots \wedge \mathrm{e}^{a_{2 i-1}} \wedge \cdots \wedge \mathrm{e}^{a_{2 n-1}} \wedge \mathrm{e}^{q}\right)\right\lrcorner w .
\end{array}
$$

\section{$3.4(2 n+1)$-gon and 2n-simplex}

We are going to prove that matrices $A^{(q)}$ given by (44) give solution to $(2 n+1)$ gon equation, by using rows of multivectors $\phi^{i, j}$. We would like to explain this in terms of representing the $(2 n+1)$-gon relation diagrammatically, like in Figure 1. The input and output $\phi$ 's are thus thought of as placed on the "legs" coming from 


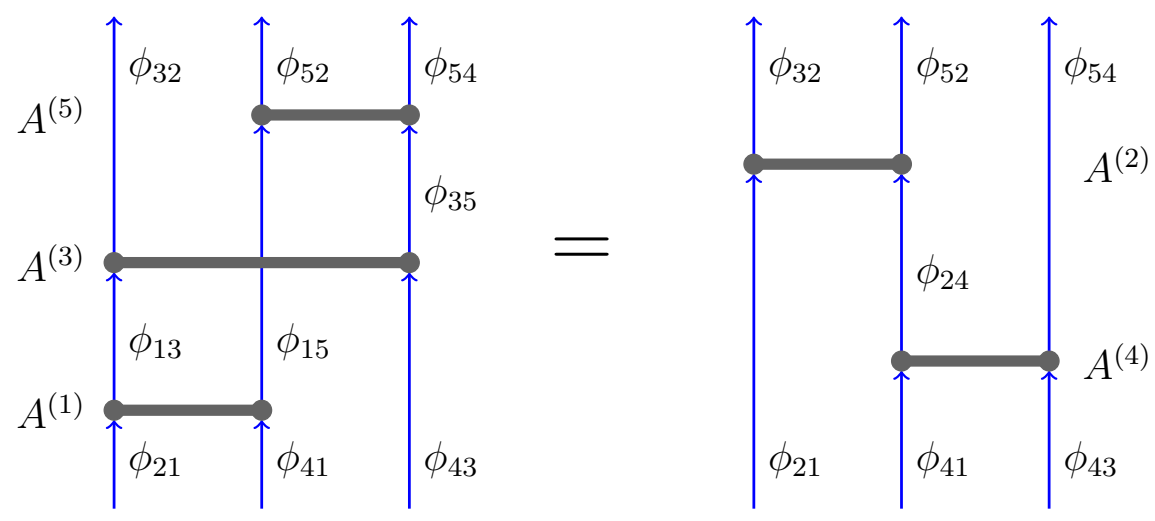

Figure 3: Multivectors $\phi^{i, j}$ as row entries for pentagon equation

one matrix $A^{(q)}$ to another, or "initial" or "final" legs, corresponding to "input" and "output" row entries for the whole l.h.s. or r.h.s.

We write, in the l.h.s., $\phi^{i, j}$ at the leg going from $A^{(i)}$ to $A^{(j)}$, while in the r.h.s., we write $\phi^{j, i}=-\phi^{i, j}$ at such leg. And in the case if the leg of $A^{(i)}$ is "initial" or "final", the missing index $j$ is taken from the matrix having the corresponding "initial" or "final" leg in the other side of the equation.

It is important that, due to the antisymmetry of $\phi^{i, j}$ in $i$ and $j$, this agrees with (46) (just change the order of indices of $\phi$ in the r.h.s. of (46), because the corresponding legs go out of $A^{(q)}$, and the minus in (46) disappears, as desired).

For $B^{(q)}$, we write $\phi$ 's in the very same way, and this again agrees with (48).

We will also prove in this Subsection that our $A^{(q)}$ and $B^{(q)}$ give a solution of $2 n$-simplex equation (via (28) ). In that proof, we will be using rows of $\phi$ 's in the same fashion as above, but also we will be using columns of $\psi$ 's.

Example 6. The case yielding matrices $A^{(q)}$ for pentagon equation is given in Figure 3

Theorem 1. $(2 n+1)$-gon equation (15) is satisfied by matrices $A^{(q)}$ (44).

Proof. Draw the diagrammatic representation of the $(2 n+1)$-gon equation, and write a $\phi^{i, j}$ at each edge as explained above. Then it turns out that:

- the $n(n+1) / 2$ input $\phi$ 's are the same for the l.h.s. and r.h.s.,

- they are linearly independent due to Proposition (9),

- and l.h.s. and r.h.s. gave the same output $\phi$ 's.

Hence, l.h.s. $=$ r.h.s.

It follows, of course, that (12) is satisfied by $B^{(q)}(45)$. 
Theorem 2. $2 n$-simplex equation is satisfied by $R$-matrices (28), with $A^{(q)}$ as in (44) and $B^{(q)}$ as in (45).

Proof. First, we note that our matrices $R^{(q)}$ of type (28), and with $B^{(q)}=$ $\left(A^{(q)}\right)^{-1}$, are obviously equal to their inverses:

$$
R^{(q)}=\left(R^{(q)}\right)^{-1} .
$$

It follows immediately that the r.h.s. of the $2 n$-simplex equation (15) is equal to $(\text { l.h.s. })^{-1}$. We have thus to prove that

$$
\text { 1.h.s. }=(\text { l.h.s. })^{-1} \text {. }
$$

Moreover, as we noted after Proposition 4, the 1.h.s. is a direct sum of matrices corresponding, first, to the red and blue sectors, and second, to the green sector. And there is no problem with blue and red sectors: they give, essentially, the same $(2 n+1)$-gons already proved in Theorem 1 (alternatively, (50) in the blue and red sectors is clear from the block matrix form (301)). It remains thus to prove (50) only for the green sector submatrix.

First, we consider the case where our field $F$ is not of characteristic 2: char $F \neq 2$. In this case, we will prove that the l.h.s. in the green sector is a diagonalizable matrix with eigenvalues only \pm 1 (which obviously implies (501)).

We will do it as follows: first, using row vectors made of our $\phi$ 's (41), we will show the existence of at least $\frac{n(n+1)}{2}$-dimensional row eigenspace with eigenvalue +1 , then, using column vectors made of our $\psi$ 's (42), we will show the existence of at least $\frac{n(n-1)}{2}$-dimensional row eigenspace with eigenvalue -1 . This will clearly give us what we want, because together we get

$$
\frac{n(n+1)}{2}+\frac{n(n-1)}{2}=n^{2},
$$

which is, as one can easily check, the full dimension of the green sector. Moreover, this will show of course that the dimensions of the mentioned eigenspaces are exactly $\frac{n(n+1)}{2}$ and $\frac{n(n-1)}{2}$.

First, we put the $\phi$ 's on edges in the same manner as in the proof of Theorem 1 , and refer to Proposition 10 (i). This gives (at least) a $n(n+1) / 2$-dimensional linear row eigen(sub)space to +1 , as desired.

Second, we put the $\psi$ 's on edges in almost the same manner as $\phi$ 's, but adding minuses where needed. This is because of different signs in the right-hand sides of (47) and (49) compared to (46) and (48). Namely, we put minuses at the input row entries of $R^{(j)}$ with an odd $j$, or/and output row entries of $R^{(k)}$ with an even $k$, and pluses otherwise. As one can check, this brings no contradictions because of Proposition 5 (ii), and the signs agree with (47) and (49). Hence, we have, according to Proposition 10 (ii), (at least) an $n(n-1) / 2$-dimensional linear column eigen(sub)space to -1 . 
Finally, we note that the case char $F=2$ follows easily from the case char $F=0$. Namely, as soon as we have proved that $R$-matrices made of matrices (44) and (45) satisfy the $2 n$-simplex equation (15) in characteristic 0 , and taking into account that (15) can be re-written as a system of polynomial identities with integer coefficients (and matrix $\mathcal{M}$ (40) entries as variables), we obtain (15) in characteristic 2 simply by the reduction modulo 2 from characteristic 0 .

Example 7. For illustration of how the $\phi$ 's and $\psi$ 's are placed on the 1.h.s. of the 4-simplex equation diagram, see Figures 4 and 5.

Example 8. It is instructive to examine the "trivial" case $n=1$ : the trigon equation [4] and the 2-simplex (Yang-Baxter) equation. Matrix $\mathcal{M}$ (40) representing an element of Grassmannian $\operatorname{Gr}(2,3)$ is then

$$
\mathcal{M}=\left(\begin{array}{ccc}
\alpha_{1} & \alpha_{2} & \alpha_{3} \\
\beta_{1} & \beta_{2} & \beta_{3}
\end{array}\right)
$$

and we find

$$
A^{(1)}=-\frac{p_{1,3}}{p_{1,2}}, \quad A^{(2)}=\frac{p_{2,3}}{p_{1,2}}, \quad A^{(3)}=-\frac{p_{2,3}}{p_{1,3}}
$$

the trigon equation is

$$
A^{(1)} A^{(3)}=A^{(2)} .
$$

The analogue of Figure 1 looks as follows:

$$
\text { lhs: } \quad 12 \stackrel{A^{(1)}}{\longrightarrow} 13 \stackrel{A^{(3)}}{\longrightarrow} 23, \quad \text { rhs: } \quad 12 \stackrel{A^{(2)}}{\longrightarrow} 23 .
$$

Formula (28) is reduced to $R^{(q)}=A_{1}^{(q)} B_{2}^{(q)} P_{12}$, hence

$$
R^{(1)}=\left(\begin{array}{cc}
0 & -\frac{p_{1,3}}{p_{1,2}} \\
-\frac{p_{1,2}}{p_{1,3}} & 0
\end{array}\right), \quad R^{(2)}=\left(\begin{array}{cc}
0 & \frac{p_{2,3}}{p_{1,2}} \\
\frac{p_{1,2}}{p_{2,3}} & 0
\end{array}\right), \quad R^{(3)}=\left(\begin{array}{cc}
0 & -\frac{p_{2,3}}{p_{1,3}} \\
-\frac{p_{1,3}}{p_{2,3}} & 0
\end{array}\right),
$$

which is a trivial solution of the entwining [17] Yang-Baxter equation (2):

$$
R_{12}^{(1)} R_{13}^{(2)} R_{23}^{(3)}=R_{23}^{(3)} R_{13}^{(2)} R_{12}^{(1)}
$$

On the other hand, we can interpret the upper index as a parameter. For example setting

$$
\mathcal{M}=\left(\begin{array}{ccc}
1 & -\mu & 0 \\
0 & -\lambda & 1
\end{array}\right), \quad R(\lambda)=\left(\begin{array}{cc}
0 & \lambda \\
1 / \lambda & 0
\end{array}\right)
$$

we get

$$
R^{(1)}=R(\lambda), \quad R^{(2)}=R(\lambda \mu), \quad R^{(3)}=R(\mu)
$$

and equation (51) becomes

$$
R_{12}(\lambda) R_{13}(\lambda \mu) R_{23}(\mu)=R_{23}(\mu) R_{13}(\lambda \mu) R_{12}(\lambda) .
$$




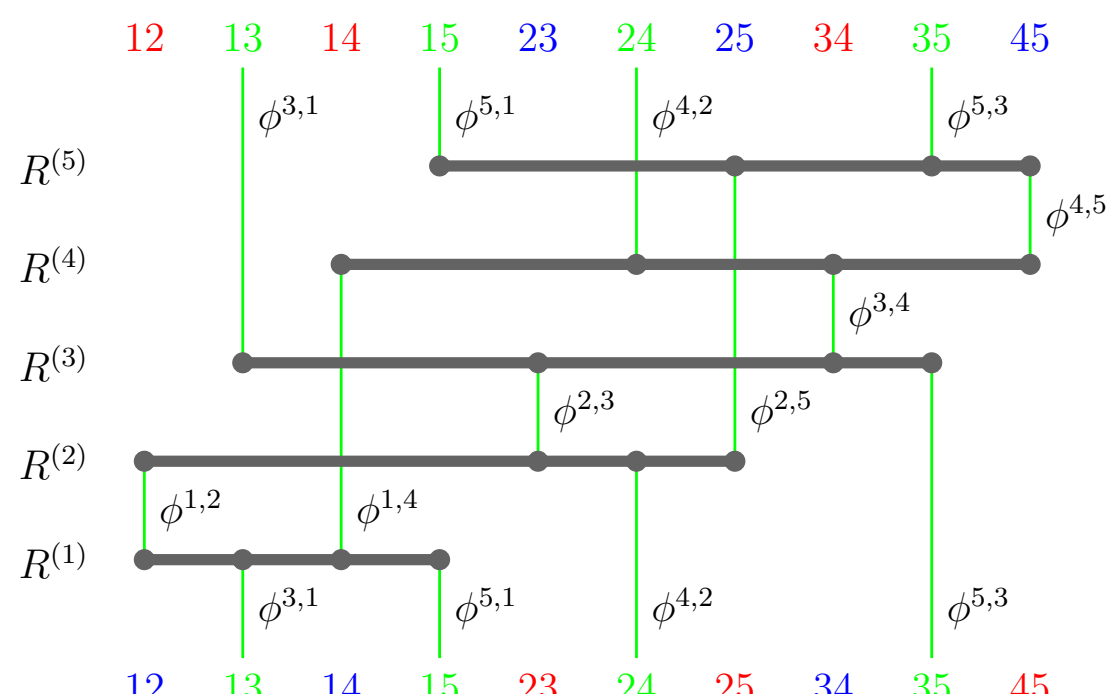

Figure 4: $\phi$ 's in the green sector of 4-simplex equation, left-hand side

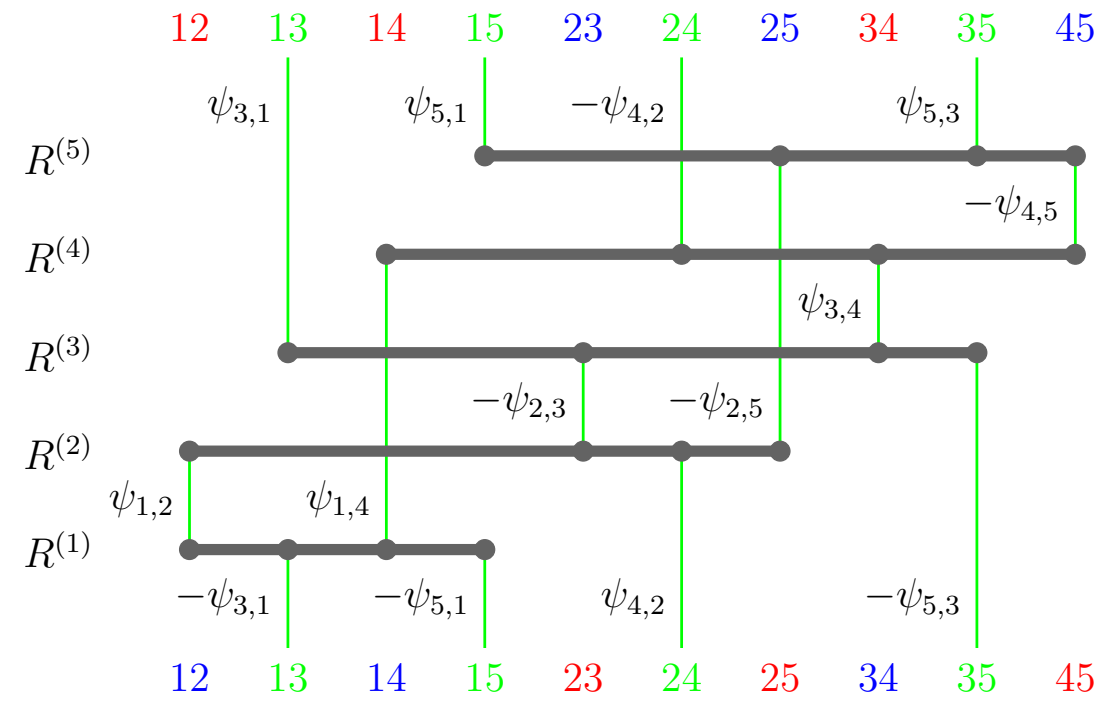

Figure 5: $\psi$ 's in the green sector of 4-simplex equation, left-hand side 


\section{Discussion}

We have constructed new "nonconstant" solutions to polygon and simplex equations, in their "direct-sum" form. Our construction has been known earlier only for the simple case of pentagon [15]. In the heptagon case, there are also different solutions [14, or at least their possible relations to the present paper is not yet known. Our results may be applied to both topology of piecewise linear manifolds and as a starting point in search for solutions of quantum equations to be used in mathematical physics. Also, it may be interesting to try and apply our nonconstant Yang-Baxter to constructing invariants of knots and knotted surfaces.

Concerning directions of further research, we can mention the following.

Our "polygons" considered here had always an odd number $(2 n+1)$ of "vertices", so we have left out of our consideration half of the possible equations. Among this half, there are certainly very interesting equations that deserve a separate research, such as a direct sum hexagon [12, 13] (note also a quantum version of hexagon in [10, Eq. (21)]).

One more interesting direction of research may be searching for noncommutative generalizations of our constructions using a division ring instead of our field $F$, and quasideterminants and quasi-Plücker coordinates [6].

And of course the natural next step consists in studying the cohomology of our solutions, as one more possible step towards constructing solutions to quantum equations. In these latter, $A^{(q)}$ or $R^{(q)}$ or their analogues are linear operators acting in the tensor product of the corresponding spaces (identified of course with their tensor products with the identities in the lacking spaces). The simplest quantum solutions are obtained from set-theoretic solutions as follows: for each $i$, consider the vector space $V_{i}$ over a field $K$ whose basis is $X_{i}$-that is, such $V_{i}$ consists of formal (finite) linear combinations

$$
\alpha x_{i}+\beta y_{i}+\ldots, \quad \alpha, \beta, \ldots \in K, \quad x_{i}, y_{i}, \ldots \in X_{i}
$$

and define, say, $R_{\text {quantum }}^{(q)}$ for Yang-Baxter requiring that

$$
\text { if } R_{i j}^{(q)}\left(x_{i}, y_{j}\right)=\left(z_{i}, t_{j}\right), \quad \text { then } \quad R_{\text {quantum }}^{(q)}\left(x_{i} \otimes y_{j}\right)=\left(z_{i} \otimes t_{j}\right) .
$$

Cohomology arises if we add multipliers to (52), that is, set

$$
R_{\text {quantum }}^{(q)}\left(x_{i} \otimes y_{j}\right)=c\left(x_{i}, y_{j}\right)\left(z_{i} \otimes t_{j}\right), \quad c\left(x_{i}, y_{j}\right) \in K^{*},
$$

where $K^{*}$ is the multiplicative group of $K$. A similar definition for other simplex or polygon equations can of course be also done; note that $K$ may be different from our field $F$, for instance, one can take $K=\mathbb{C}$, while $F$ being a finite field. More about such cohomology theories can be found in [16, 12, 13]. 


\section{References}

[1] Yasuhiro Akutsu, Tetsuo Deguchi, and Miki Wadati. The Yang-Baxter relation: a new tool for knot theory. In Braid group, knot theory and statistical mechanics, pages 151-200. World Scientific, 1989.

[2] Felix Alexandrovich Berezin. Introduction to Superanalysis. Dordrecht: D. Reidel Publishing Company, first edition, 1987.

[3] J Scott Carter and Masahico Saito. Knotted surfaces and their diagrams, volume 55 of Mathematical Surveys and Monographs. American Mathematical Soc., 1998.

[4] Aristophanes Dimakis and Folkert Müller-Hoissen. Simplex and polygon equations. SIGMA. Symmetry, Integrability and Geometry: Methods and Applications, 11:042, 2015.

[5] Aristophanes Dimakis and Folkert Müller-Hoissen. Matrix KP: tropical limit and Yang-Baxter maps. Letters in Mathematical Physics, 109(4):799-827, 2019.

[6] Israel Gelfand, Sergei Gelfand, Vladimir Retakh, and Robert Lee Wilson. Quasideterminants. Advances in Mathematics, 193:56-141, 2005.

[7] ND Gilbert and T Porter. Knots and Surfaces. Oxford University Press, 1994.

[8] Jarmo Hietarinta. Permutation-type solutions to the Yang-Baxter and other n-simplex equations. Journal of Physics A: Mathematical and General, 30(13):4757, 1997.

[9] Seiichi Kamada. Knot invariants derived from quandles and racks. Geom. Topol. Monogr., 4:103-117, 2002.

[10] Rinat Kashaev. A simple model of 4d-TQFT. In D Wood, J de Gier, C Praeger, and T Tao, editors, 2016 MATRIX Annals, volume 1 of MATRIX Book Series, pages 277-286. Springer, 2018.

[11] RM Kashaev and SM Sergeev. On pentagon, ten-term, and tetrahedron relations. Communications in mathematical physics, 195(2):309-319, 1998.

[12] Igor G Korepanov. Nonconstant hexagon relations and their cohomology. arXiv preprint arXiv:1812.10072, 2018. Accepted for publication in Lett. Math. Phys.

[13] Igor G Korepanov. Polynomial-valued constant hexagon cohomology. arXiv preprint arXiv:1904.07000, 2019. 
[14] Igor G Korepanov. Heptagon relation in a direct sum. arXiv preprint arXiv:2003.10335, 2020.

[15] Igor G Korepanov and Nurlan M Sadykov. Pentagon relations in direct sums and Grassmann algebras. Symmetry, Integrability and Geometry: Methods and Applications, Apr 2013.

[16] Igor G Korepanov, Georgy I Sharygin, and Dmitry V Talalaev. Cohomologies of $n$-simplex relations. Mathematical Proceedings of the Cambridge Philosophical Society, 161(2):203, 2016.

[17] Theodoros Kouloukas and Vassilios Papageorgiou. Entwining Yang-Baxter maps and integrable lattices. Banach Center Publications, 93(1):163-175, 2011.

[18] Sergei Matveev. Distributive groupoids in knot theory. Math. USSR Sbornik, 47:73-83, 1984.

[19] Igor Rostislavovich Shafarevich. Basic algebraic geometry, volume 1. Springer, 1994. 